\title{
Postpartum Ovarian Vein Thrombosis: Clinical Case
}

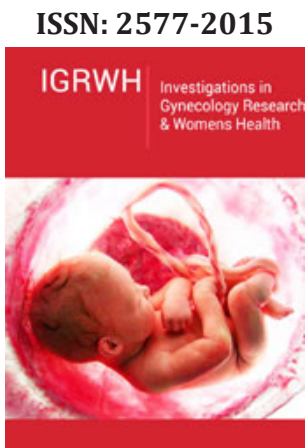

*Corresponding author: Ana Ribeiro, Department of Obstetrics and Gynecology, Centro Hospitalar do Baixo Vouga, Aveiro, Portugal

Submission: 俔 October 24, 2019

Published: 眥 December 9, 2019

Volume 3 - Issue 2

How to cite this article: Ana Ribeiro Rafael Brás, Maria Lúcia M, António Braga, Jorge Braga. Postpartum Ovarian Vein Thrombosis: Clinical Case. Invest Gynecol Res Women's Health.3(3). IGRWH.000561.2019.

DOI: 10.31031/IGRWH.2019.03.000561

Copyright@ Ana Ribeiro, This article is distributed under the terms of the Creative Commons Attribution 4.0 International License, which permits unrestricted use and redistribution provided that the original author and source are credited.

\author{
Ana Ribeiro'* ${ }^{*}$, Rafael Brás², Maria Lúcia $\mathbf{M}^{2}$, António Braga² and Jorge Braga² \\ ${ }^{1}$ Department of Obstetrics and Gynecology, Centro Hospitalar do Baixo Vouga, Aveiro, Portugal \\ ${ }^{2}$ Department of Obstetrics and Gynecology, Centro Materno Infantil do Norte, Porto, Portugal
}

\begin{abstract}
Ovarian vein thrombosis (OVT) is a rare but potentially serious postpartum complication, which occurs in $0.05 \%$ to $0.18 \%$ of pregnancies and is diagnosed on the right side in $80 \%$ to $90 \%$ of the cases $[1,2]$. As OVT can mimic acute abdomen it should be considered in the differential diagnosis of postpartum acute abdomen [3]. We present a case of a 29-year-old female who presented a diffuse abdominal pain, fever and vomit on her third postpartum day. Her computed tomography demonstrated left ovarian vein repletion defect compatible with the presence of a thrombus. She was treated with enoxaparin and antibiotics, which lead to a resolution of the thrombus. Our case highlights the importance of prompt diagnosis and treatment of OVT in order to prevent morbidity and mortality.
\end{abstract}

Keywords: Ovarian vein thrombosis; Thrombosis; Anticoagulation; Postpartum 3.

Abbreviations: OVT: Ovarian Vein Thrombosis; CT: Computed Tomography

\section{Introduction}

Ovarian vein thrombosis is a rare but potentially serious condition that affects mostly postpartum women, but it may also be associated with a variety of pelvic conditions, such as pelvic inflammatory disease, gynecological surgery, and malignancies [1]. Early recognition and treatment of this condition is needed to avoid the morbidity and the mortality related both to the thrombosis and to any associated infection or sepsis. OVT can occur in $0.02 \%$ to $0.18 \%$ of pregnancies, $80 \%$ to $90 \%$ occur in the right side [2]. This is believed to be due, in part, to the dextrorotation of the enlarging uterus that commonly occurs during pregnancy, which compresses the right ovarian vein and right ureter as they cross the pelvic rim [4]. The usual clinical symptoms are pelvic or diffuse abdominal pain, fever and right-sided palpable mass [5].

We present a case of a 29-year-old female who presented a diffuse abdominal pain, fever and vomit on her third postpartum day. Her computed tomography (CT) demonstrated left ovarian vein repletion defect compatible with the presence of a thrombus. She was treated with enoxaparin and antibiotics, which lead to a resolution of the thrombus. Our case highlights the importance of prompt diagnosis and treatment of OVT in order to prevent morbidity and mortality.

\section{Clinical Case}

A 29-year-old woman at three days postpartum of cesarean section complains of severe diffuse abdominal pain, fever, nausea and anorexia. Intestinal transit not reestablished, neither for gas. Her abdomen very distended, tympanized, with pain on superficial and deep palpation in all quadrants and missing bowel sounds. Pelvic examination showed an involuted uterus below the umbilical line. Normal mouthpieces, without smell. There was no evidence of deep vein thrombosis in the lower extremities. Laboratory exams revealed elevated white blood cell count $\left(19000 \mathrm{cel} / \mathrm{mm}^{3}\right.$ ) with neutrophilia and elevated C reactive protein $(289 \mathrm{mg} /$ dL). A transvaginal ultrasound was performed that showed a small amount of blood within the endometrial cavity and the abdominal ultrasound was unremarkable.

After excluding possible causes of acute abdomen, the patient underwent intravenous contrast-enhanced computed tomography (CT) that demonstrated left ovarian vein repletion 
defect compatible with the presence of a thrombus (Figure 1\&2). Blood cultures were positive for Escherichia coli. The patient started treatment with enoxaparin and intravenous antibiotic therapy for 5 days. She was discharged on the $10^{\text {th }}$ postpartum day. It was recommended to continue enoxaparin for at least three months, with outpatient follow-up with hematology.

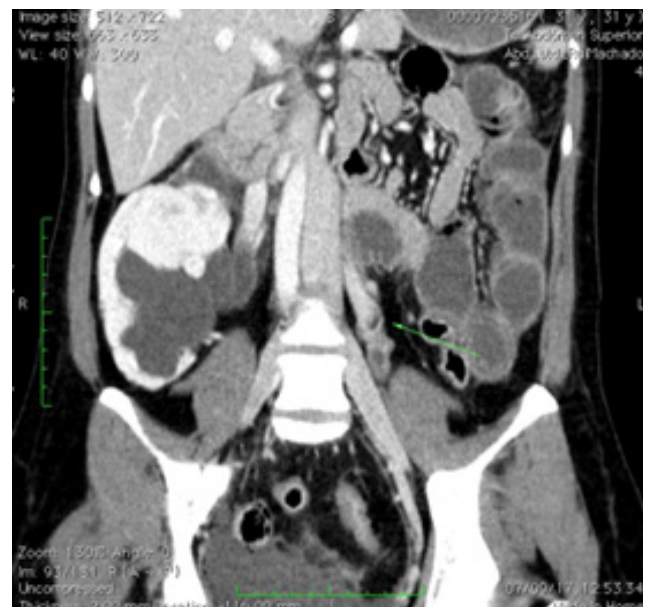

Figure 1: Coronal Demonstrates (CT) an enlarged ovarian vein with central hypodensity, representing thrombosis (arrow).

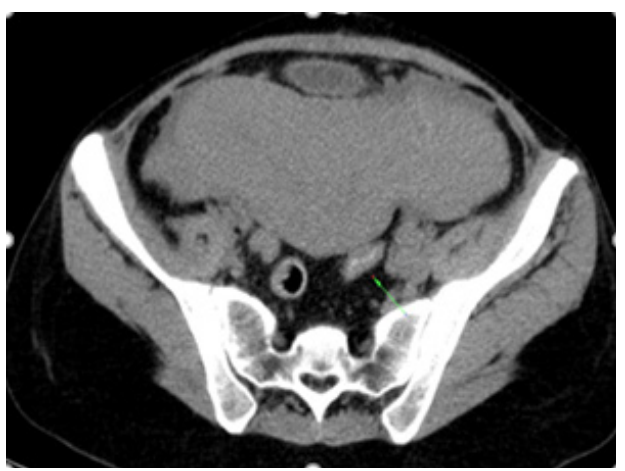

Figure 2: Abdominal CT scan-arrow showing thrombosed right ovarian vein.

\section{Discussion}

OVT is a rare condition, with a reported incidence of 0.002$0.05 \%$ in pregnancies, being more common after cesarean section $[6,7]$. Besides its association with pregnancy and puerperium, pelvic inflammatory disease, malignancy and gynecological surgery also represent risk factors [8,9]. In $70-90 \%$ of cases involves the right side which was different from our reported clinical case. It is hypothesized that OVT commonly occurs on the right side because the right ovarian vein is longer than the left and it lacks competent valves. The right ovarian vein enters the inferior vena cava at an acute angle, which makes it more susceptible to compression $[10,11]$. Furthermore, the dextrorotation of the enlarged uterus that occurs in pregnancy can cause compression of the right ovarian vein and the right ureter as they cross the pelvic rim, which causes stasis of blood leading to thrombosis [10]. During the puerperium, there is anterograde flow in the right ovarian vein as compared to the retrograde flow in the left ovarian vein, which predisposes to rightsided thrombosis. These facts are in accordance with the Virchow's triad of vessel wall injury, venous stasis and hypercoagulability that ascribe the pathophysiology of OVT $[8,12]$.
OVT prompt diagnosis is important to prevent morbidity and mortality and should be considered in the clinic of pelvic or flank pain, abdominal palpable mass, fever and elevated inflammatory parameters [11].However these symptoms are also suggestive of other diseases, such as appendicitis, pyelonephritis, urinary tract infection, adnexal torsion, puerperal endometritis and tubo-ovarian abscess which can delay the OVT diagnosis [13]. The diagnosis is made based on the clinical features. Magnetic resonance angiography has the highest sensitivity and specificity but CT scan with intravenous contrast can also be used. Normally the CT scan shows a thick-walled enlarged ovarian vein with rim enhancement and central hypodensity $[14,15]$.

The main complication is pulmonary embolism (25\%) which results in death in $5 \%$ of cases. So inf OVT is not diagnosed, the disease could extend into the inferior vena cava or iliofemoral vessels and lead to pulmonary embolization. Other complications include ovarian abscess, ovarian infarction, uterine necrosis and sepsis [16]. The recommended treatment is intravenous antibiotic therapy and anticoagulation although there are no specific guidelines for the duration of treatment $[17,18]$. In the 
case presented here, the patient presented as risk factors for VTE, previous pregnancy and cesarean section.

\section{Conclusion}

OVT is a rare condition and can be associated with serious complications if left untreated. High index of suspicion is required for the prompt diagnosis and management especially in cases that mimic acute abdomen.

\section{References}

1. Ortin X, Ugarriza A, Espax RM (2005) Postpartum ovarian vein thrombosis. Thromb Haemost 93(05): 1004-1005.

2. Dunnihoo DR, Gallaspy JW, Wise RB, Otterson WN (1991) Post-partum ovarian vein thrombophlebitis: A review. Obstet Gynecol Surv 46(7): 415-427.

3. Kubik HRA, Hebisch G, Huch R, Hilfiker P, Debatin JF, et al. (1999) Role of duplex color Doppler ultrasound, computed tomography, and MR angiography in the diagnosis of septic puerperal ovarian vein thrombosis. Abdom Imaging 24: 85-91.

4. Sinha D, Yasmin H, Samra JS (2005) Postpartum inferior vena cava and ovarian vein thrombosis-A case report and literature review. J Obstet Gynaecol 25(3): 312-313.

5. Jenayah AA, Saoudi S, Boudaya F (2015) Ovarian vein thrombosis. Afr Med J 21: 251.

6. Kominiarek MA, Hibbard JU (2006) Postpartum ovarian vein thrombosis: An update. Obstet Gynecol Surv 61(5): 337-342.

7. Royo P, Burgos A, García M, Lecumberri R, Alcázar JL (2008) Postpartum ovarian vein thrombosis after cesarean delivery: A case report. J Med Case Reports 2: 105.

8. Salomon O, Apter S, Shaham D (1999) Risk factors associated with postpartum ovarian vein thrombosis. Thromb Haemost 82(3): 10151019.
9. Harris K, Mehta S, Iskhakov E, Chalhoub M (2012) Ovarian vein thrombosis in the nonpregnant woman: An overlooked diagnosis. H Ther Adv Hematol 3: 325-328.

10. Derrick FC, Rosenblum RR, Lynch KM (1967) Pathological association of the right ureter and right ovarian vein. J Urol 97(4): 633-640.

11. Calderwood CJ, Jamienson R, Greer IA (2007) Gestational related changes in the deep venous system of the lower limb on light reflection rheography in pregnancy and the puerperium. Clin Radiol 62: 11741179.

12. Nikolaos A, Dionysios D, Anneza Y (2011) Ovarian vein thrombosis mimicking acute abdomen: A case report and literature review. World J Emerg Surg 6: 45.

13. Johnson A, Wietfeldt ED, Dhevan V, Hassan I (2010) Right lower quadrant pain and postpartum ovarian vein thrombosis. Uncommon but not forgotten. Arch Gynecol Obstet 281(2): 261-263.

14. Quane LK, Kidney DD, Cohen AJ (1998) Unusual causes of ovarian vein thrombosis as revealed by CT and sonography. AJR Am J Roentgenol 171: 487-490.

15. Twickler DM, Setiawan AT, Evans RS (1997) Imaging of puerperal septic thrombophlebitis: Prospective comparison of MR imaging, CT, and sonography. AJR Am J Roentgenol 169: 1039-1043.

16. Clarke CS, Harlin SA (1999) Puerperal ovarian vein thrombosis with extension into the inferior vena cava. Am Surg 65: 147-150.

17. Själander A, Jansson JH, Bergqvist D, Eriksson H, Carlberg B, et al. (2008) Efficacy and safety of anticoagulant prophylaxis to prevent venous thromboembolism in acutely ill medical inpatients: A meta-analysis. J Intern Med 263(1): 52-60.

18. Salomon 0, Dulitzky M, Apter S (2010) New observations in postpartum ovarian vein thrombosis: experience of a single center. Blood Coagul Fibrunolsys 21: 16-19. 